\title{
On the Comparison of IoT Layers Along with the Applications and Intellectual Property Threats
}

\author{
Sourav Rana ${ }^{1}$, Tripti Sharma ${ }^{2}$, Veenu Grover ${ }^{3}$, Yeshu Sharma ${ }^{4}$ \\ ECE Department, IPEC, Ghaziabad, India
}

\begin{abstract}
In this paper, we have given a brief introduction about IOT. A discussion over the Architecture of IOT has been done according to the OSI model with a tabular comparison among the layers. Then Scopes and Application of IoT has been discussed including some major projects. Every Technology is proven to be a boon as well as bane so this Research papers also lighten up some threats that humankind experiences because of IoT.
\end{abstract}

Keywords: IoT, Physical layer, Datalink Layer, Network Layer, Transport Layer, Session Layer, Presentation layer, Application layer, Home security model, Intellectual Property Theft, Brand Damage

\section{Introduction}

The term Iot was first introduced by Kevin Ashton in 1998, is the future of internet and deals with the future of connectivity and reachability. It may be technically defined as a "System of interrelated computing devices, mechanical and digital machines, objects, animals or people that are provided with uniqueidentifiers and the ability to transfer data over a network without requiring human-to-human or human-to-computer interaction. "[1] .

In IoT, 'things' refer to any object on face of the Earth, whether it is a communicating device or a noncommunicating object. IoT has evolved from the convergence of wireless technologies, microelectromechanical systems (MEMS), microservices and the internet which allows unstructured machine-generated data to be analyzed for insights that will drive improvements[2]. Iotenables devices/objects to observe, identify and understand a situation or the surroundings without being dependent on human help.in Industrial front IoT acts a transformational force that improves a company's performance, efficiency, asset utilization by IoT Analytics.

\section{IoT Layers Per OSI Model}

\subsection{Physical Layer}

This layer is responsible for electrical, mechanical or procedural checks. In this data, will be converted into binary i.e., 0 and 1 . The devices working at physical layer are cables, hubs, modems, etc[3].

\subsection{Datalink Layer}

This layer is divided into two sublayers -

1) Logical link control (LLC)

2) Media access control (MAC)

The devices working on datalink layer are switch, bridge, NIC, etc. The LLC talks about WAN protocols like HDLC, whereas MAC is used for physical address.

\subsection{Network Layer}

This layer is responsible for providing best path to data to reah the destination. The device that works on network layer is Router. The network layer is divided in two parts -

1) Routed Protocols

2) Routing Protocols

The logical addressing is a part of network layer.

\subsection{Transport Layer}

This layer is responsible for end to end connectivity. It is considered as heart of OSI layers. The various tasks performed by this layer are

1) Multiplexing and Demultiplexing

2) Segmentation

3) Sequencing and Reassembling

\subsection{Session Layer}

This layer is responsible for establishing, managing and terminating the connections between the application. It sets up, coordinates and terminates conversation's, exchanges and dialogues between the applications. It may be stated with the examples such as RPC(Remote procedure call) and SQL(Structured Query Language)

\subsection{Presentation Layer}

It converts data into standard format providing independence from differences in data representation by translating from application to network format. It provides freedom from compatibility problem and So, it is also called as SYNTAX LAYER. It performs following tasks:-

1) Encoding-Decoding

2) Encryption -Decryption

3) Compression-Decompression

\subsection{Application Layer}

It supports applications and end user processes.It is responsible for providing network services to the user and is also called as DESKTOP LAYER. Services are distributed according to the port number which vary up to 65535 . These ports are nothing but sockets that is exit point to the layer. 


\section{International Journal of Science and Research (IJSR) \\ ISSN (Online): 2319-7064}

Index Copernicus Value (2015): 78.96 | Impact Factor (2015): 6.391

Here $0-1023$ are the Reserved Ports whereas 1024 to 65535 are the Open ports. Now, a comparison is drawn between these layers on the basis of their difference in features and their Application area.

Table 1: Comparison between OSI Layers [4]

\begin{tabular}{|c|c|c|c|}
\hline Sno. & Layers & Features & Applications \\
\hline 1. & $\begin{array}{l}\text { Physical } \\
\text { Layer }\end{array}$ & $\begin{array}{c}\text { In this data is } \\
\text { converted into binary. }\end{array}$ & $\begin{array}{c}\text { Network adaptors, fibre } \\
\text { media convertors, } \\
\text { repeaters etc. }\end{array}$ \\
\hline 2. & $\begin{array}{l}\text { Data Link } \\
\text { Layer }\end{array}$ & $\begin{array}{l}\text { It is used to determine } \\
\text { physical addresses. }\end{array}$ & $\begin{array}{l}\text { For error correction and } \\
\text { flow control. }\end{array}$ \\
\hline 3. & $\begin{array}{l}\text { Network } \\
\text { Layer }\end{array}$ & $\begin{array}{l}\text { It provides best path } \\
\text { for the data to reach } \\
\text { the destination. }\end{array}$ & $\begin{array}{c}\text { Multicast group } \\
\text { management, network } \\
\text { layer information and } \\
\text { error. }\end{array}$ \\
\hline 4. & $\begin{array}{l}\text { Transport } \\
\text { Layer }\end{array}$ & $\begin{array}{l}\text { It is used for end to } \\
\text { end connectivity. }\end{array}$ & $\begin{array}{l}\text { Multiplexing and } \\
\text { demultiplexing. }\end{array}$ \\
\hline 5. & $\begin{array}{l}\text { Session } \\
\text { Layer }\end{array}$ & $\begin{array}{l}\text { It is responsible for } \\
\text { establishing, } \\
\text { maintaining and } \\
\text { terminating the } \\
\text { session. }\end{array}$ & $\begin{array}{l}\text { Application environments } \\
\text { that use RPC (Remote } \\
\text { Procedure Call). }\end{array}$ \\
\hline 6. & $\begin{array}{l}\text { Presentation } \\
\text { Layer }\end{array}$ & $\begin{array}{l}\text { It converts the data } \\
\text { into standard format. }\end{array}$ & $\begin{array}{l}\text { Serialisation of objects } \\
\text { and other data structures, } \\
\text { conversion of an } \\
\text { EBCDIC-coded text file } \\
\text { to an ASCII coded file. }\end{array}$ \\
\hline 7. & $\begin{array}{l}\text { Application } \\
\text { Layer }\end{array}$ & $\begin{array}{c}\text { It contains all services } \\
\text { needed by OS to } \\
\text { communicate on the } \\
\text { network. }\end{array}$ & $\begin{array}{c}\text { Identification of } \\
\text { communication partners, } \\
\text { determination of resource } \\
\text { availability and } \\
\text { synchronization of } \\
\text { communication. }\end{array}$ \\
\hline
\end{tabular}

\section{Scope of IoT}

\subsection{IoT and Networking}

The internet of things requires a network which can 2 handle increased demand for data analytics, agility and security. Without any network to support there will be no internet of things. Sensors and gadgets will gather increasingly large amounts of data. But the internet of things is about more than just gadgets and displays. In this, the amount of data gathered will seriously impact the network and networking industry needs to find out the possible implications [5].

The areas of IoT that will impact the network are:

*Data analytics

*Agile networking

*Security

\subsection{IoT and Embedded Systems}

The increasing growth of IoT solutions create a vast new opportunity for developers of embedded systems. These opportunities bring with them a lot of challenges. The developers of embedded systems used to build devices that allow seamless connectivity with one another. They used to address security risks to protect highly interconnected, often wireless systems from intrusion while ensuring performance and reliability. End to end IoT solution enables the ability to develop and manage embedded systems safely and securely [6].

\subsection{IoT and Smart City}

People continue to move to cities for a lot of different reasons, such as employment opportunities, lifestyle, career and more. As this migration continues, cities will need to become more efficient and improved in order to keep up with the increasing population. Thus, smart cities will start to become the norm in the major metropolitan areas of the world. Therefore, smart cities use internet of things (IoT) devices such as connected sensors, lights and meters to collect and analyse the data to improve infrastructure, public utilities and services and more[7].

\section{Applications of IoT}

\subsection{Traffic Congestion Monitoring and Management}

The problem of traffic is rising as the use of vehicles is increasing day by day. Traffic congestion is the problem which is faced due to more traffic increasing day by day. IoT devices such as sensors can help to simply overcome the problem of traffic congestion. The system designed to solve this problem with the help of IoT, consists of an IR sensor which is placed at some distance from the traffic lights. This IR sensor will detect the traffic on the road and will then classify that traffic with a label as light, normal or heavy traffic. The assumption of the traffic by the IR sensor will be taken with the help of a microcontroller and a GPRS module installed in the system [8].

\subsection{Transport and Logistics}

It is one of the first business sectors interested in IoT technologies. Under this, currently two systems are already available and deployed: ConLock and Contain Safe. It is the integration of light sensors, GPS and GSM[9].

\subsection{Healthcare}

The internet of things is about to change the way we live and work. And if it reaches its fullest potential, it will change every aspect of our lives. This kind of change is evident in the healthcare sector, where the pen and paper has been the primary means of recording patient's information for decades. But now, healthcare technology is changing in major ways through IoT[10].

\subsection{IoT based Automated Parking System}

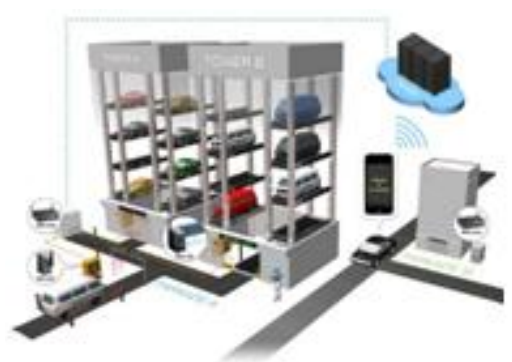

Figure 1: Automated Parking System

Parking being an important issue in India Modern Automated Parking system give a head start to the solution of this problem. Itincludes integrated electromechanical 


\section{International Journal of Science and Research (IJSR) \\ ISSN (Online): 2319-7064}

Index Copernicus Value (2015): 78.96 | Impact Factor (2015): 6.391

transmission control, security control, detection systems and automated placement. It helps in vehicle measurement, image analysis, electronic payment scanning, and automatic retrieval. Under this automated parking complex Images of the car are captured and transmitted to a controller where dimensions of the cars are read and the license plate are recorded. While, the driver pays by card and the system provides authorization. The car will be moved by an elevator up to the storage floors and parked into an appropriate space with security guarantee[11].

IOT provides us a gateway to connect with the PLC (Programmable Logic Controller) and the sensors; along with the field data can be retrieved, analyzed and stored. It helps to link the PLC with the PC-based monitoring control system, allowing PLC data to be read and basic repair and maintenance tasks executed by way of a handheld panel.

The Schematic representation of the automated parking system is depicted with apt. personification of each.

- Raspberry pi: It is a credit-card sized microcontroller, now available in its latest version of Raspberry Pi3 , is used to implement the parking system and it is attached with Raspberry pi camera, which captures the picture of the parking area to validate the slots.

- Navigation system: It signals the availability of parking slots to the users and navigates to the exact location of nearest parking area from current location.

- Display device: It is the monitor or tab that is used to display the admin side interface and allow him to modify the parking lots by observing the device.

- User device: By this the user can connect with the smart parking system with their smart phones or with some browsers.

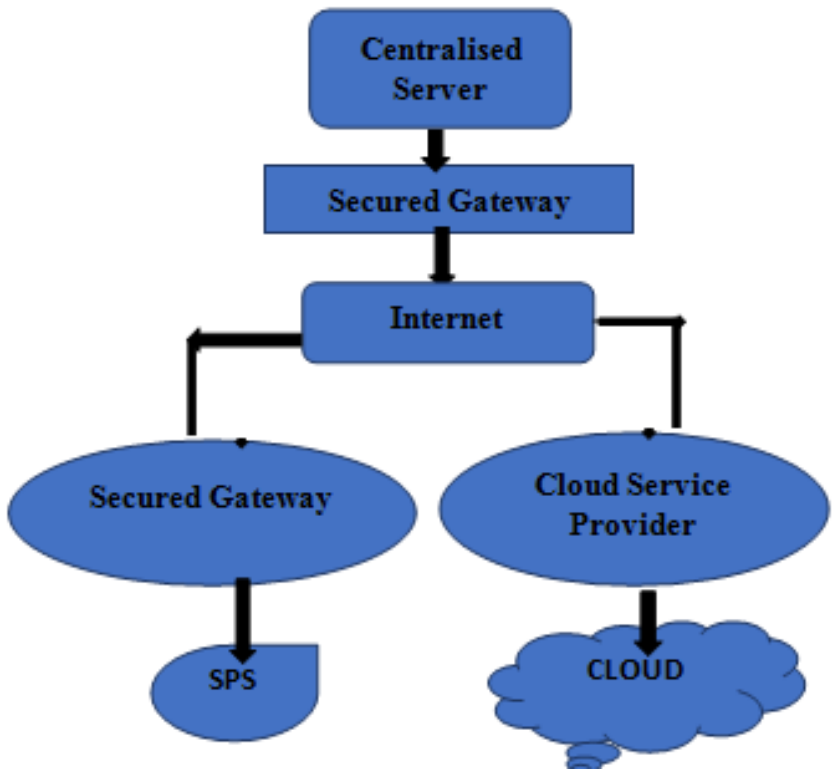

Figure 2: Flow Chart of Automated Parking System[12]

It isn't easy as it sounds, many challenges were faced during process because the automated parking garages represent a significant investment, connection of existing PLC systems and equipment to external facilities requires a reliable, costeffective IoT solution which can make easy to upload field data to the cloud with no need to abandon established assets with least investment and reduced maintenance burden.

\subsection{IoT Home Security Model}

The IoT Home Security Model can be accessed by using any smart device and PC to monitor the security status within the house through the data collected from sensors through the Internet, with the requirement of a few hardware tools such as Arduino Yun, sensors and LEDs etc. When the homeowner turn on the security mode, any recognized harmful activities that occurred within the house will be detected through installed sensors and automatically forward the alert message to the homeowner through the internet. The data from sensors will constantly be stored in the cloud storage and interact with the custom web-based application that enables it to display the data in a website, and this help the homeowner to have an advantage to monitor any potential harmful activity that might occur within the house in real-time. Another additional feature of security in this project is Access Control Technology, this system uses the (NFC) "Near Field Communication" approach to provide access to the recognized individuals (guests or family members) with the rights to enter the house[13].

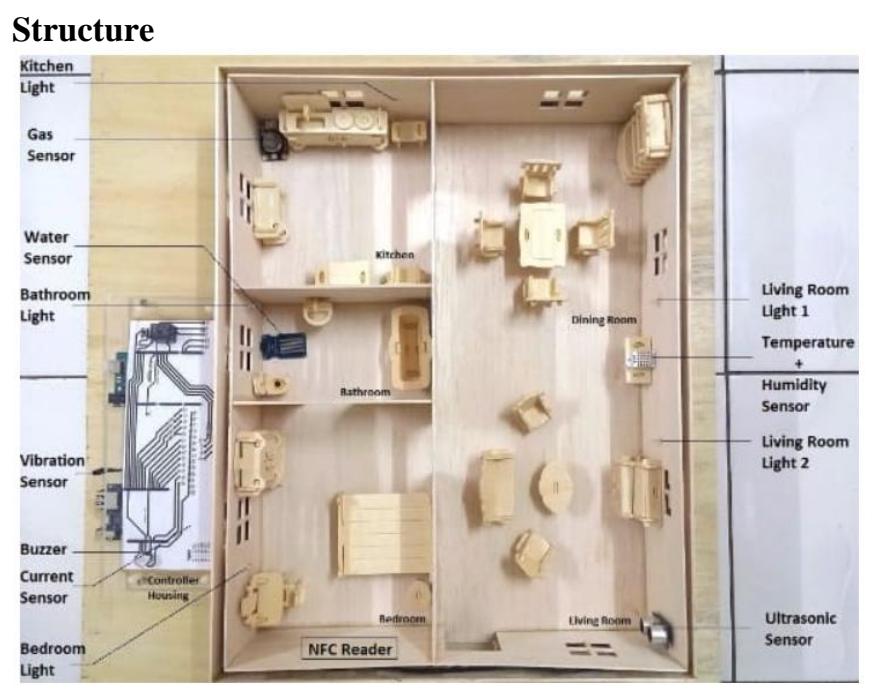

Figure 3: Structure of Home Security System [14]

The structure of this model is to build per the floor plan layout. The main electronic parts consist of 6 sensors, 1 NFC Reader and 2 microcontrollers, other minor components can refer to my components list and the materials that used in this structure are plywood with size $64.5 \mathrm{~cm}(\mathrm{~L}) \times 61.5 \mathrm{~cm}$ (W) $\times 0.8 \mathrm{~cm}(\mathrm{H})$ as the ground, craft plywood sheets for building the walls. Thus, the finishing product is almost $85 \%$ covered by plywood material.

\section{IoT Threats}

\subsection{Intellectual Property Theft}

It has been estimated that the cyber theft of intellectual property in American companies is similar in scale to that of America's total export to Asia. Per FBI investigation this theft has increased around $50 \%$ this year. The impact at a single hacked manufacturer Codan which is an Australian company that sells metal detectors and mining technology, had its blueprints for metal detector stolen, per Reuters [15]. 


\section{International Journal of Science and Research (IJSR) \\ ISSN (Online): 2319-7064}

Index Copernicus Value (2015): 78.96 | Impact Factor (2015): 6.391

\subsection{Brand Damage}

After 30 years of the Bhopal gas tragedy in which thousands of people died and many were injured, the Dow Chemical Company is still suffering brand damage. Because brand damage, the International Olympic Committee got criticized by London Assembly for its Dow Chemical sponsorships based on their links to the tragedy. The various causes of the incident included operating errors, design flaws, temperature and level sensors prevented the early detection of the incident [16].

\section{Conclusion}

In this paper, we analysed the various solutions that are currently available for the implementation of urban IoT's. The discussed technologies are very close to being standardized, and industry players are already active in the production of devices that take advantage of these technologies to enable the applications of interest. Future developments in the Internet of Things will optimize the information flow in industrial and social scenarios and revolutionise business and private communication. Like other milestones in technology the Internet of Things enables us to measure what we could not do before. This paper also covered some possible threats of IoT. The proposed architecture is just one possible solution, but it is based on existing developments. In fact, while the range of design options for IoT systems is rather wide, the set of open and standardized protocols is significantly smaller. The enabling technologies, furthermore, have reached a level of maturity that allows for the practical realization of IoT solutions and services, starting from field trials that will hopefully help clear the uncertainty that still prevents a massive adoption of the IoT paradigm.

\section{Acknowledgement}

This study about IoT was supported by IPEC. We thank our faculty from the ECE department, who provided us the insight and expertise that greatly assisted the study.

\section{References}

[1] M. Rouse "Internet of things", TechTarget, IOT Agenda

[2] M. Rouse "Internet of things", TechTarget, IOT Agenda

[3] V. Beal "The 7 layers of the OSI model", 1999

[4] V. Beal "The 7 layers of the OSI model", 1999

[5] P.G. Menon, "IoT: The network impact", Network Computing, 2014

[6] T.Angelucci, "Embedded systems and the internet of things- What's under the hood?", ORACLE, 2014

[7] A..Zanella, N.Bui, A.Castellani, L.Vangelista, M.Zorzi, "Internet of things for smart cities" IEEE Internet of Things Journal( Volume: 1, Issue: 1, Feb. 2014 )

[8] Techpacs.com , "Project description of traffic congestion monitoring and management"

[9] P. Puzio, "Internet of things and its applications", 2011

[10] A.Meola, "Internet of things in healthcare: Information technology in health", 2016

[11] ADLINK, "IoT based automated parking systems"
[12] Basavaraju S R , “Automatic smart parking system using internet of things" IJSR Publications, Volume 5, Issue 12, 2015

[13] A.Kow, "IoT Home security model”, 2016

[14] A.Kow, "IoT Home security model", 2016

[15]B.Kaye and J.Wardell, "Australian metal detector company counts cost of Chinese hacking”, 2015

[16]F.Bermingham, "Years on from Bhopal gas tragedy, Dow Chemicals shareholders demand justice", International Business Times, 2014 